\title{
Inputs to the dorsal striatum of the mouse reflect the parallel circuit architecture of the forebrain
}

\author{
Weixing X. Pan', Tianyi Mao ${ }^{2}$ and Joshua T. Dudman ${ }^{\text {* }}$ \\ Janelia Farm Research Campus, Howard Hughes Medical Institute, Ashburn, VA, USA \\ 2 Vollum Institute, Oregon Health Sciences University, Portland, OR, USA
}

Edited by:

Jose L. Lanciego, University of

Navarra, Spain

Reviewed by:

Jose L. Lanciego, University of

Navarra, Spain

María Elena Erro Aguirre, Servicio

Navarro de Salud, Spain

${ }^{*}$ Correspondence:

Joshua T. Dudman, Janelia Farm

Research Campus, 19700 Helix Drive,

Ashburn, VA 20147, USA.

e-mail:dudmanj@janelia.hhmi.org
The basal ganglia play a critical role in the regulation of voluntary action in vertebrates. Our understanding of the function of the basal ganglia relies heavily upon anatomical information, but continued progress will require an understanding of the specific functional roles played by diverse cell types and their connectivity. An increasing number of mouse lines allow extensive identification, characterization, and manipulation of specified cell types in the basal ganglia. Despite the promise of genetically modified mice for elucidating the functional roles of diverse cell types, there is relatively little anatomical data obtained directly in the mouse. Here we have characterized the retrograde labeling obtained from a series of tracer injections throughout the dorsal striatum of adult mice. We found systematic variations in input along both the medial-lateral and anterior-posterior neuraxes in close agreement with canonical features of basal ganglia anatomy in the rat. In addition to the canonical features we have provided experimental support for the importance of non-canonical inputs to the striatum from the raphe nuclei and the amygdala. To look for organization at a finer scale we have analyzed the correlation structure of labeling intensity across our entire dataset. Using this analysis we found substantial local heterogeneity within the large-scale order. From this analysis we conclude that individual striatal sites receive varied combinations of cortical and thalamic input from multiple functional areas, consistent with some earlier studies in the rat that have suggested the presence of a combinatorial map.

Keywords: striatum, retrograde, tracing, basal ganglia, mouse

\section{INTRODUCTION}

In the mammalian telencephalon four interconnected nuclei comprise the basal ganglia: the striatum, the globus pallidus (GP), the subthalamic nucleus (STN), and the substantia nigra (SN; Haber, 2003). Pathological or experimental disruptions of neuronal activity in the basal ganglia cause profound deficits in motor control and learning throughout the chordate phylum, from amphibians to primates (Reiner et al., 1998). The vast majority of excitatory projections enter the basal ganglia via the striatum (Tepper et al., 2007). As a result, the circuitry of the striatum in vertebrates has been the subject of intense study since the pioneering application of techniques for tract tracing by Nauta and colleagues in the middle of the twentieth century (Paxinos, 2004).

It was observed in the earliest tract tracing studies that axons from subregions and lamina of the neocortex were inhomogeneously distributed in the dorsal striatum of rats (Gerfen, 1984) or the caudate-putamen $(\mathrm{CPu})$ of primates (Goldman and Nauta, 1977). Careful registration of labeling patterns across subjects and the development of techniques for simultaneous use of multiple labels provided clear evidence for a topographical organization of the corticostriatal projection (Malach and Graybiel, 1986; Gerfen, 1992). Input to the dorsal striatum follows the relative position of neurons within the neocortex such that projection neurons from more lateral and dorsal cortical structures projecting to more lateral and dorsal aspects of the striatum (Paxinos, 2004). In addition to the corticostriatal projection, it has also been demonstrated that the thalamostriatal and nigrostriatal projections are topographically organized (Haber, 2003; Paxinos, 2004; Haber and Calzavara, 2009). In addition, anterograde tracing has demonstrated that the topographic organization of the striatum is maintained in a more compact form at its output targets in the ventral midbrain (Tulloch et al., 1978; Deniau et al., 2007).

The profound effects of pathological disruptions have indicated a critical role for the processing of excitatory input within the striatum (Albin et al., 1989). The topographical organization of inputs to and outputs from the striatum has long played a central role in both theory and the interpretation of functional data in the basal ganglia (Ragsdale and Graybiel, 1990; Mink, 1996). The core functional property of the basal ganglia from this perspective is the processing of information in largely non-overlapping "loops" that connect functionally related regions of the cortex, basal ganglia, and thalamus (Graybiel et al., 1994; Haber, 2003). An important support for this model was a series of elegant studies in the monkey that revealed a convergence of functionally related, but anatomically distant, neocortical regions (Flaherty and Graybiel, 1991, 1995; Parthasarathy et al., 1992; Parthasarathy and Graybiel, 1997). However, it is not clear that the convergence of functionally related cortical regions is a general feature across vertebrates. For example, functional mapping studies (Brown, 1992; Brown and Sharp, 1995; Brown et al., 1996, 1998) and anatomical tracing experiments (Alloway et al., 2000, 2006; Hoffer and Alloway, 2001; Ramanathan et al., 2002; Hoffer et al., 2005) have revealed that 
sensory and motor cortical regions project to diverse targets within the rodent striatum. While convergence is clearly present, it may be diminished compared to that detected in primates with $<5-10 \%$ of overlap in functionally related somatosensory and motor corticostriatal projections (Hoffer and Alloway, 2001).

Although the extent and importance of functional convergence is unclear in rats, and unknown in mice, in the last several years it has become clear that anatomically distinct regions of the striatum have dissociable functional properties (Packard and Knowlton, 2002; Balleine et al., 2009 Redgrave, 2010 \#256). It has been elegantly demonstrated in rats that a profound dissociation between goaldirected and habitual behavior could be mapped onto circuits in the dorsomedial (Yin et al., 2005a,b) and dorsolateral striatum (Yin et al., 2006), respectively. More recently, an analogous dissociation between the dorsomedial and dorsolateral striatum during motor learning has also been demonstrated in mice (Yin et al., 2009). These recent behavioral results have shed light on the critical and specific roles of the dorsal striatum in instrumental conditioning and motor control that complement and extend earlier ideas about roles of striatal subregions in modality specific learning (Graybiel et al., 1994; Packard and Knowlton, 2002). However, these results in rodents raise the question of whether a finer scale organization of specific cortical and thalamic inputs is present in addition to the large-scale separation of medial and lateral input patterns.

The above mentioned differences between the anatomy of the dorsal striatum in rats and primates highlights the need for careful anatomical studies in different species. Despite deep similarities in the anatomy of the basal ganglia in vertebrates, significant differences are nonetheless present (Reiner et al., 1998). At best, analogies between closely related species (such as the rat and the mouse) need to be transformed into precise anatomical localizations in each. At worst, the failure of such analogies has the potential to cause misinterpretation of functional data. Yet, despite the substantial literature on the vertebrate striatum, there have been relatively few anatomical studies of the macrocircuitry of the mouse (White and DeAmicis, 1977; Hersch and White, 1982; Porter and White, 1983; Bernardo and Woolsey, 1987; Mattiace et al., 1989; Hofstetter and Ehret, 1992; Cepeda et al., 2003; Miura et al., 2007; Brazhnik et al., 2008; Usunoff et al., 2009; Ibáñez-Sandoval et al., 2010; Tlamsa and Brumberg, 2010) and to our knowledge an examination across multiple topographic regions has not been previously conducted.

To further establish the relationship between defined anatomical circuits and behavior we suggest that a detailed accounting of the similarities and differences between the anatomy of the mouse striatum and other vertebrates will be of continued importance. As an initial attempt at creating a more comprehensive anatomical description of the basal ganglia in mice, here we analyze a series of injections of retrograde tracer throughout the dorsal striatum. We focus on the topographic organization of the dorsal striatum and its connectivity with both cortical and subcortical structures.

\section{MATERIALS AND METHODS PREPARATION AND STEREOTACTIC INJECTION OF FLUORESCENT LATEX MICROSPHERES}

A total of 35 male mice aged 2-4 months were used. All work involving animals was approved by the Janelia Farm Institutional Animal Care and Use Committee (Protocol Number 08-36), and met the standards of the Association for Assessment and Accreditation of Laboratory Animal Care, International (AAALAC) accredited program. Mice were housed in ventilated cage racks (Allentown, Inc), provided ad lib irradiated feed (Lab Diet 5053), reverse osmosis water chlorinated to 2-3 ppm, and were kept on a 12:12 light cycle. For the duration of the surgical exposure of the brain, implantation of cannulae, and intracranial injections mice were maintained at a surgical plane of anesthesia using isoflurane $\left(1.5-2.5 \%\right.$ in $\mathrm{O}_{2}$ ). In several injections, after the mouse was deeply anesthetized and secured in the stereotaxis, guide cannulae spanning the depth of the cortex were implanted in order to minimize the leak of fluorescent beads during insertion of the injection needle into the brain. For guide cannulae we used one or two sterile, 30 gage (26 gage in some cases) stainless-steel guide cannula. After lowering the guide cannula to the appropriate depth dental cement was used to secure the cannulae to the skull.

Injections were targeted to a range of coordinates centered around (1) posterior, ( -0.2 posterior, 2.5-2.8 lateral, 2.5-3.5 ventral; all units are $\mathrm{mm}),(2)$ medial anterior $(+1.0$ anterior, 1.2 lateral, 2.5-3.5 ventral), and (3) lateral anterior (+1.0 anterior, 2.5 lateral, 2.5-3.5 ventral) striatal targets. In some experiments injections were targeted to the motor $(+1.0$ anterior, 0.7 lateral, $0.4-0.7$ ventral) and somatosensory cortical areas $(-0.55$ posterior, 3.0 lateral, $0.4-0.7$ ventral). A fine needle ( $140 \mu \mathrm{m}$ external diameter and $40 \mu \mathrm{m}$ internal diameter) made by silica capillary tubing (VS-140-40, Scientific Glass Engineering) was used as injection needle, which was held by an attached stainless-steel collar. The tip of needle extended 1-2 mm beyond the tip of guide cannula. The needle was filled with red or green Lumafluor beads (Lumafluor beads, Lumafluor, Inc.) or a mixture of both and mated to a $1 \mu \mathrm{l}$ Hamilton syringe with plastic tubing. Following implantation of the needle $80-100 \mathrm{nl}$ of beads were infused into the target areas with the speed of $100 \mathrm{nl} / \mathrm{min}$ using a microinjection driver. The needle was left in place for 10 min before being removed from the brain.

\section{PREPARATION AND STEREOTACTIC INJECTION OF VIRUSES}

Stereotactic injection of viruses was accomplished using procedures equivalent to those described above for injection of retrograde tracer. The virus was a modified adeno-associated virus (serotype 2/1) engineered to drive the over expression of enhanced green fluorescent protein (eGFP). The sequence for the expression construct and details of viral production are described in more detail in subsequent manuscript (Mao et al., in preparation). Injection volumes for virus were $20-40 \mathrm{nl}$ per injection site with 1-2 sites per target region.

\section{HISTOLOGY}

In initial experiments we found that similar results were obtained after allowing axonal transport of the retrograde tracer for as few as 3 days or as many as 14 days after injection. For the experiments reported here, 3-7 days following tracer injection and 1-3 weeks following viral infection, mice were deeply anesthetized under constant inhalation of isoflurane $\left(>2 \%\right.$ in $\left.\mathrm{O}_{2}\right)$ and transcardially perfused with $4 \%$ paraformaldehyde in phosphate buffered saline $(0.1 \mathrm{M}, \mathrm{pH}=7.4)$. The brains were removed and left in a $4 \%$ paraformaldehyde solution for 1-7 days for the injection of retrograde tracers or $24 \mathrm{~h}$ for viral infection. Each brain was sectioned on a 
vibrating microtome at 50-100 $\mu \mathrm{m}$. The sections were mounted onto slides and allowed to air dry. Preliminary examination of labeling was accomplished using a dissecting microscope equipped with filter sets for epifluorescence detection (Olympus MVX10). Images were obtained using acquisition with a digital camera or in a small number of cases using a Zeiss LSM710 confocal microscope.

\section{ANALYSIS}

For mapping of the injection sites and cell labeling areas, the photos of slices were checked through. The injection and labeling sites were determined according to a standard stereotaxic atlas (Paxinos and Franklin, 2004). To do quantitative analyses, an ideal method is to count the percentage of all labeled cells in a given brain region area. However, this analysis is complicated by a challenge in defining a brain region as well as the challenge of producing high quality stereological counts of all cells in a brain area. Finally, we find that the signal-to-noise of labeled cells can vary widely in some regions (especially the neocortex) and thus, the definition of a positive labeled cell is not rigorous. We also found that it was impossible to accurately define the exact extent or magnitude of the injection site. Thus, here we settled upon a semi-quantitative method to quantify labeling in a given brain region. For a particular labeled region, the relative intensity of labeling of an area was defined by counting the numbers of labeled somata. The intensity marker " + " was give for the numbers of labeling cells were counted as 1-10, “++" for 11-20, " +++ " for 21-30, and " ++++ " for more than 31.

Tabulated results from manual annotation and counting were analyzed using custom written routines in Igor Pro (Wavemetrics, Eugene, OR, USA) and Matlab (Mathworks, Natick, MA, USA). Correlation and covariance analysis was accomplished using standard Matlab functions applied to our annotated database of all labeling intensity. All plotting and curve fitting was accomplished using custom scripts written for Igor Pro.

\section{RESULTS}

Retrograde tracers were injected under stereotaxic control into the dorsal striatum of mice using volume displacement (Figure 1). Our injection sites were distributed throughout the dorsal striatum with particular focus on the less studied posterior striatum. In preliminary experiments we tested both the injection of latex microspheres adsorbed with fluorescent small molecules (Lumafluor beads, Lumafluor, Inc.) and high molecular weight dextrans conjugated with fluorescent small molecules (10,000 MW, Invitrogen). For a given injection of tracer, labeled somata were found in relatively extensive regions of the neocortex, and more focal regions of the thalamus and midbrain as early as 2 days following injection into the dorsal striatum. We found that latex microspheres ("beads") gave more reliable labeling with improved signal-to-noise and thus all of the data reported here will focus on our injections using beads. We found qualitatively similar results using both tracers, however, a detailed comparison was not conducted.

\section{QUALITATIVE DESCRIPTION OF LABELED SOMATA}

As expected, the majority of labeled somata were found in the neocortex, discrete nuclei in the thalamus, and nuclei of the ventral midbrain reflecting the corticostriatal, thalamostriatal, and nigros- triatal pathways, respectively (Figure 1). The complete distribution of labeled somata, an indication of the maximal cell count, and the frequency of detection (see Materials and Methods for details) from all of the injections of fluorescent beads examined are collected in Figure 2A and Table 1. All abbreviations follow the conventions of the standard mouse brain atlas (Paxinos and Franklin, 2004). Consistent with canonical descriptions of the striatal circuitry, we found these three pathways to have core features that are shared between primates (Haber, 2003) and rodents (Paxinos, 2004). One, labeled somata were found in deep layers of a wide distribution of frontal and parietal neocortex (Figures 1 and 3). Two, the intralaminar nuclei of the thalamus were a major source of neurons projecting to the striatum (Figure 4). Three, the dopaminergic input to the dorsal striatum originated from neurons with somata located predominantly in the substantia nigra pars compacta $(\mathrm{SNc})$, but also found in the ventral tegmental area (VTA) and the retrorubral field of the caudal midbrain (A8; Figure 5).

In our dataset we regularly observed more intensely labeled and larger numbers of cortical neurons in the ipsilateral hemisphere as compared to the contralateral hemisphere. Additionally, posterior and lateral injections regularly yielded less contralateral labeling than more anterior and medial injections. As a result the quantification of labeled subregions of cortex and their quantification were derived entirely from ipsilateral projections. While labeled cortical somata were confined almost exclusively to deep layers, there were substantial apparent differences in the distribution of labeled somata between frontal cortices, temporal cortices, and sensory areas of parietal cortices. In well-defined six-layer neocortex labeled somata were clearly confined to distributions of cells throughout the entire extent of layer $\mathrm{V}$ and often concentrated in both a narrow superficial band consistent with layer Va and deeper layers (Figures $\mathbf{1}$ and $\mathbf{3}$ ). However, in frontal and temporal cortices labeled neurons appeared distributed throughout a wider range of cortical layers extending from the border of layer III to layer VI (Figure 3); reminiscent of the broad laminar distribution corticostriatal neurons identified with anterograde tracing in the frontal cortex of the rat (Gerfen, 1989).

In the thalamus we reliably observed retrograde labeling of neurons not only in the parafascicular (PF) and centromedian (CM) intralaminar nuclei (Smith et al., 2004), but also within sensory nuclei located more laterally such as the posterior (PO), ethmoid (Eth), medial (MG), and lateral geniculate (LG) nuclei (Figure 4). Across the entire collection of retrogradely labeled somata we found that individual injection sites have both idiosyncratic and focal distributions of somata in the thalamus (Table 1; Figures 2 and 4 ).

Injection sites throughout dorsal striatum were associated with intensely labeled somata in the basolateral (BLA) and central (CEA) nuclei of the amygdala (Figure 6). Our labeling was consistent with early studies in which injection of tritiated amino acids into the CEA and BLA revealed labeled axons across a large extent of the dorsal striatum in the rat (Kelley et al., 1982; Price, 1986). In addition to an amygdalostriatal projection, there has been considerable interest in the interaction between the extended amygdala and striatum. Although to our knowledge there have been no prior report of projections from the extended amygdala to the dorsal 

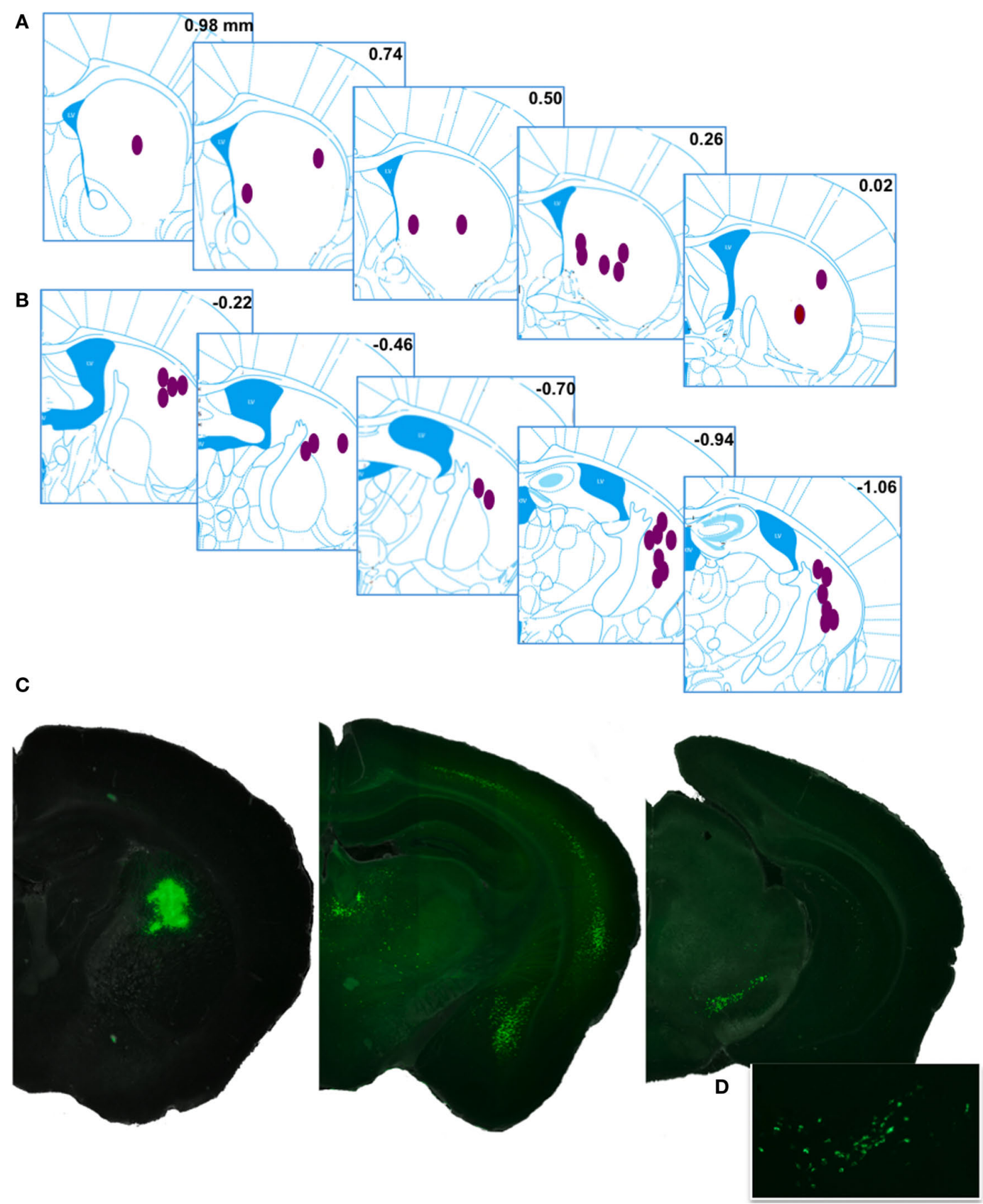

FIGURE 1 | Injection sites and representative retrograde labeling. (A,B) The approximate central points for reported injections are indicated by ellipses. Inset numbers are position $(\mathrm{mm})$ of the coronal plane relative to bregma, Anterior and posterior injection sites are displayed in $(\mathbf{A}, \mathbf{B})$, respectively. Histological references are modified from Paxinos and Franklin, 2004. (C) An example injection site with major planes of labeled somata. (D) Higher magnification fluorescence image showing the typical punctuate labeling in the ventral midbrain. striatum we found clear labeling of somata in the interstitial nucleus of the posterior limb of the anterior commissure (IPAC) at many anterior injection sites (Figure 6). Finally, there have been a series of previous studies focused on the projection from midline brainstem nuclei to the striatum (Di Matteo et al., 2008). Consistent with these results we found clear labeling of large somata in medial and dorsal raphe nuclei of the brainstem, a putative serotonergic projection to the dorsal striatum (Figure 7).
ANTEROGRADE TRACING CONFIRMS A NON-CANONICAL PROJECTION Although retrograde labeling of more lateral thalamic nuclei such as PO was previously observed in the rat (Erro et al., 2002; Alloway et al., 2006), we were nonetheless concerned that such labeling could result from leakage of some tracer in the neocortex during the insertion of the injection needle. Moreover, as described above, we also observed retrograde labeling in areas of the amygdala and midbrain that, while these sites have also been observed 


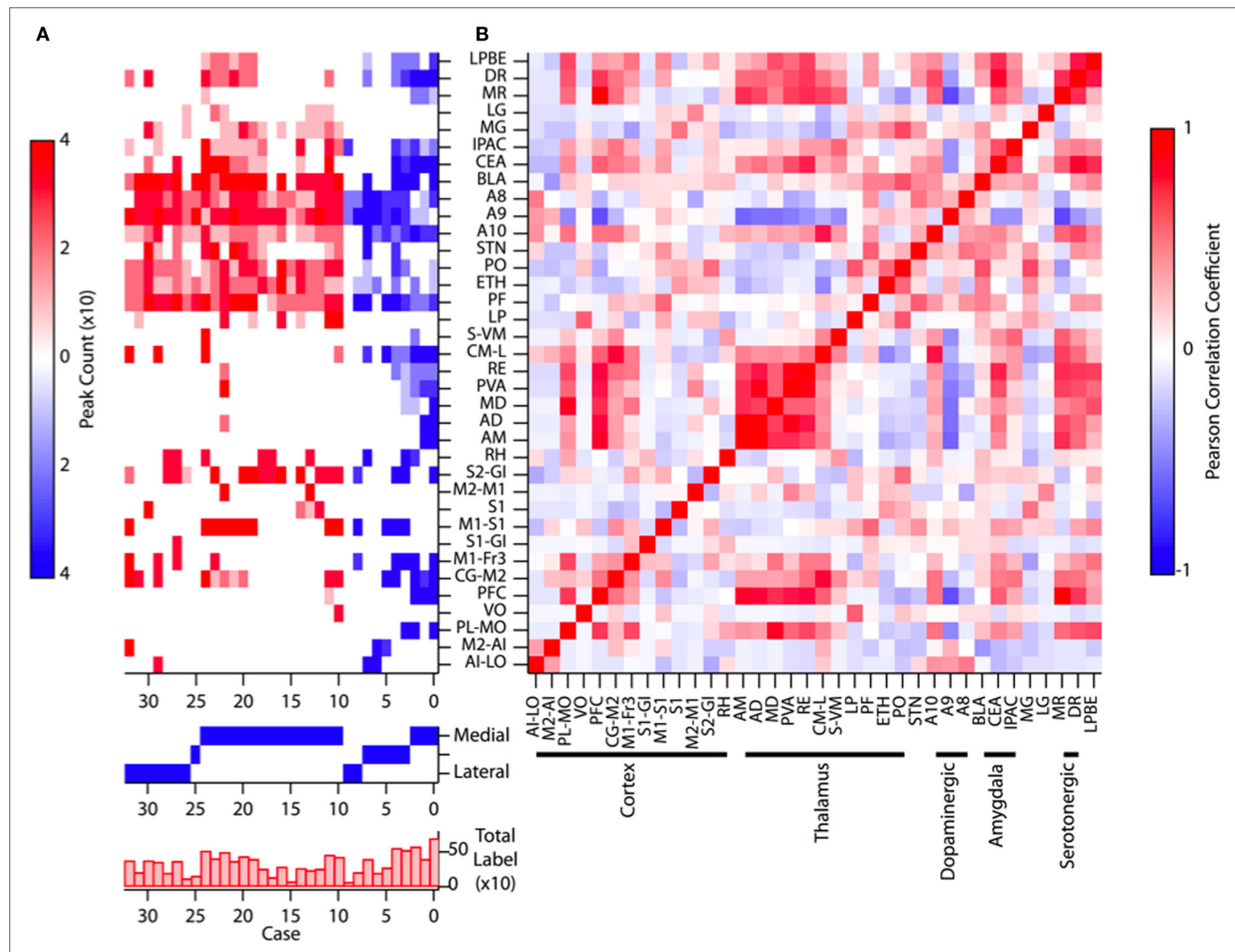

FIGURE 2 | Summary of all injections of retrograde tracer [(A), upper] Plot of labeling intensity for each injection site ("case") with peak count represented by the color scale at left. Labels for each manually annotated brain area (after Paxinos and Franklin, 2004) are indicated along the y axis. Cases plotted in blue are the most anterior injections and cases plotted in red are derived from the more posterior injections. [(A), middle] The approximate medial to lateral position of the injection in each case is indicated by a solid blue square. [(A), lower] The sum of labeling across all brain areas is plotted for each case. (B) The complete pairwise correlation matrix for all injections and all annotated brain areas. Annotated brain areas are grouped according to their principal features along the $x$ axis. For select correlation values see text. In this and subsequent figures brain region annotations follow the conventions of Paxinos and Franklin, 2004. previously, were less commonly reported in the literature. We took two steps to ensure the accuracy of our labeling. One, we found that implanting cannulae spanning the neocortex and largely prevented leakage of tracer during insertion and did not change our observed retrograde labeling in the thalamus and cortex (an example is shown in Figure 3C). Two, we sought to directly confirm the thalamostriatal projection from the more lateral nuclei using anterograde tracing.

To visualize the projection from the PO nucleus of the thalamus we infected neurons with an adeno-associated virus driving the expression of the gene encoding enhanced green fluorescent protein (GFP). Two weeks after infection expression of the GFP was sufficient to detect both somata localized to the thalamus as well as clear axonal projections coursing through the internal capsule and terminating in the cortex (Figure 8). As expected, the vast majority of axons from the posterior and ventral posterior thalamus formed a dense columnar plexus of axonal ramifications in layer IV of the neocortex (Figure 8B). However, with careful examination we found that axon collaterals from the thalamus exited the internal capsule and ramified in a band in the posterior dorsolateral striatum (Figures 8C,D) consistent with our retrograde labeling. Axons observed in the striatum appeared both branched and lined with varicosities consistent with the formation of local synaptic contacts (Figures 8D,E). The massive labeling of fibers terminating in the cortex compared with those in the striatum are consistent with sparse retrograde labeling we observed (Figure 4) and with the suggestion that only a subpopulation of neurons in lateral, posterior thalamic nuclei project to the striatum (Erro et al., 1999, 2001; BarrosoChinea et al., 2008). 
Table 1 | Summary of retrograde labeling results.

\begin{tabular}{|c|c|c|c|}
\hline $\begin{array}{l}\text { Labeling } \\
\text { sites }\end{array}$ & $\begin{array}{l}\text { Anterior-medial } \\
\text { injections }(n=4)\end{array}$ & $\begin{array}{l}\text { Anterior-lateral } \\
\text { injections }(n=8)\end{array}$ & $\begin{array}{l}\text { Posterior } \\
\text { injections } \\
(n=21)\end{array}$ \\
\hline \multicolumn{4}{|c|}{ FOREBRAIN } \\
\hline PrL-MO & $+++(2 / 4)$ & $++++(1 / 8)$ & $-(0 / 21)$ \\
\hline LO-Al & $-(0 / 4)$ & $++++(2 / 8)$ & $-(0 / 21)$ \\
\hline PrL-IL & $++1+(3 / 4)$ & $-(0 / 8)$ & $-(0 / 21)$ \\
\hline Cg1-M2 & $+++(4 / 4)$ & $++++(2 / 8)$ & $-(0 / 21)$ \\
\hline M1-Fr3 & $++++(2 / 4)$ & $++++(3 / 8)$ & $+++(4 / 21)$ \\
\hline M1-S1 & $+++(1 / 4)$ & $++++(4 / 8)$ & $++++(8 / 21)$ \\
\hline S2-GI & $+++(1 / 4)$ & $++++(3 / 8)$ & $+++(13 / 21)$ \\
\hline EcT-PRh & $+++(1 / 4)$ & $+++(3 / 8)$ & $++(4 / 21)$ \\
\hline IPAC & $++(4 / 4)$ & $++(3 / 8)$ & $++(11 / 21)$ \\
\hline \multicolumn{4}{|c|}{ AMYGDALA } \\
\hline CEA & $++1+(4 / 4)$ & $++++(2 / 8)$ & $++(10 / 21)$ \\
\hline BLA & $+++(2 / 4)$ & $+++(3 / 8)$ & $++++(18 / 21)$ \\
\hline \multicolumn{4}{|c|}{ THALAMUS } \\
\hline AM & $++++(2 / 4)$ & $-(0 / 8)$ & $-(0 / 21)$ \\
\hline AD-IAD & $++++(2 / 4)$ & $-(0 / 8)$ & $+(1 / 21)$ \\
\hline $\mathrm{MD}$ & $+++(2 / 4)$ & $+(1 / 8)$ & $-(0 / 21)$ \\
\hline PVA & $+++(3 / 4)$ & $+(1 / 8)$ & $+(1 / 21)$ \\
\hline $\mathrm{RE}$ & $++(3 / 4)$ & $+(1 / 8)$ & $-(0 / 21)$ \\
\hline $\mathrm{CM}-\mathrm{CL}$ & $+++(4 / 4)$ & $++++(4 / 8)$ & $+++(3 / 21)$ \\
\hline AM-AV & $+++(1 / 4)$ & $-(0 / 8)$ & $-(0 / 21)$ \\
\hline Sub-VM & $+++(2 / 4)$ & $-(0 / 8)$ & $-(0 / 21)$ \\
\hline LP & $-(0 / 4)$ & $++(1 / 8)$ & $++(8 / 21)$ \\
\hline PF & $+++(4 / 4)$ & $+++(6 / 8)$ & $+++(20 / 21)$ \\
\hline PO & $+(2 / 4)$ & $++(3 / 8)$ & $+++(20 / 21)$ \\
\hline Eth & $+(4 / 4)$ & $+(4 / 8)$ & $++(19 / 21)$ \\
\hline MG & $-(0 / 4)$ & $-(0 / 8)$ & $+(14 / 21)$ \\
\hline LG & $-(0 / 4)$ & $-(0 / 8)$ & $+(6 / 21)$ \\
\hline \multicolumn{4}{|c|}{ MIDBRAIN } \\
\hline A10 & $+++(4 / 4)$ & $+++(6 / 8)$ & $++(19 / 21)$ \\
\hline A9 & $+(2 / 4)$ & $++++(8 / 8)$ & $+++(21 / 21)$ \\
\hline A8 & $+++(2 / 4)$ & $+++(8 / 8)$ & $++(20 / 21)$ \\
\hline \multicolumn{4}{|c|}{ BRAIN STEM } \\
\hline DRV & $++1+(4 / 4)$ & $+++(2 / 8)$ & $++(7 / 21)$ \\
\hline $\mathrm{MnR}$ & $++(4 / 4)$ & $-(0 / 8)$ & $-(0 / 21)$ \\
\hline LPBE & $++(3 / 4)$ & $++(3 / 8)$ & $++(5 / 21)$ \\
\hline
\end{tabular}

\section{ORGANIZATION OF STRIATAL INPUTS IDENTIFIED BY RETROGRADE LABELING}

Manual inspection of the annotated database of labeled cells revealed clear tendencies in the retrograde labeling data that were partially described above. In addition, when individual injections were sorted according to the relative anterior-posterior position (blue and red coloring in Figure 2A, respectively) and secondarily sorted by their relative medial-lateral position (lower panel, Figure 2A) of injection site, clustering of the retrograde labeling was apparent. Previous work has demonstrated both a segregation of afferent inputs according to relative position along the mediallateral neuraxis as well as a convergence of functionally related, anatomically distant brain regions. While this has most often been
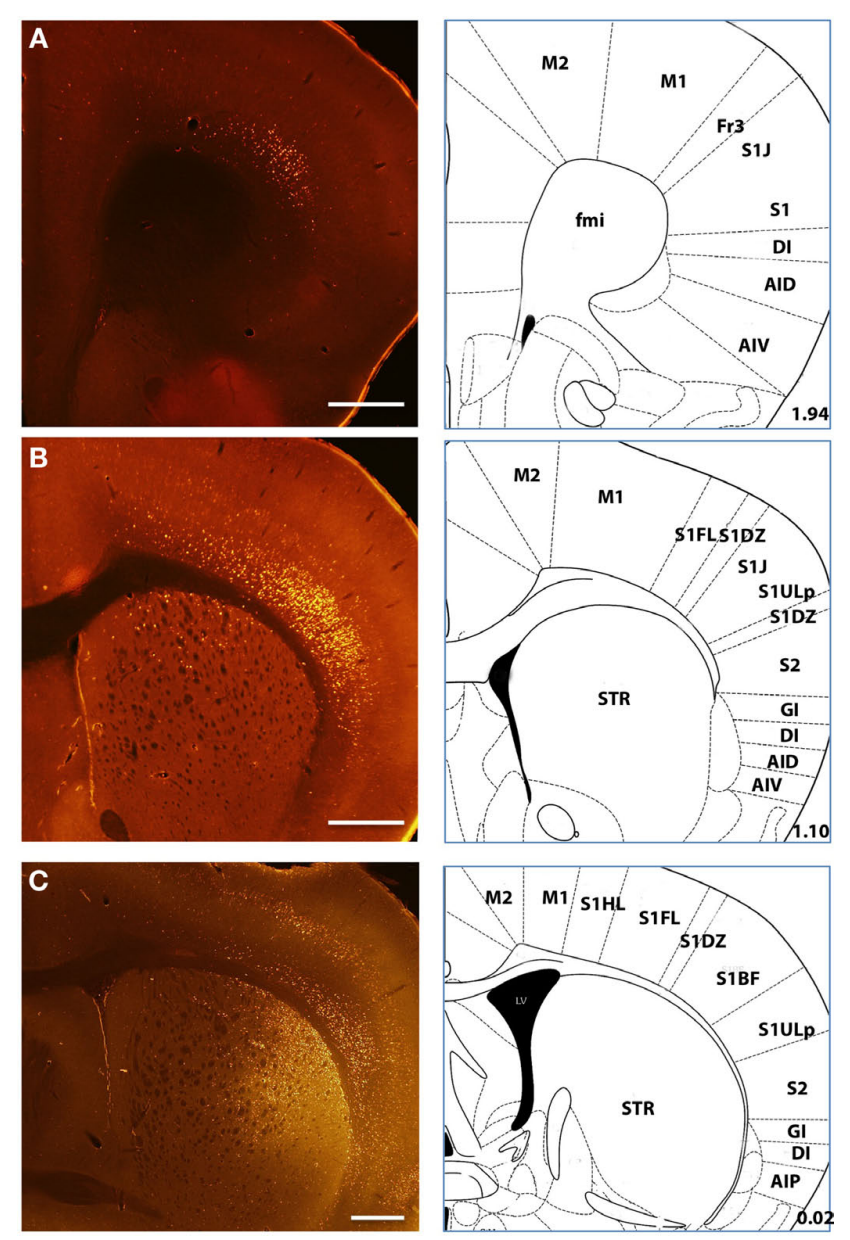

FIGURE 3 | Example labeling of deep layer cortical neurons Labeled neurons following retrograde tracer injections into the dorsal striatum showed labeling distributed over a range of sensory and motor cortical regions (A-C). Intense labeling of both superficial and deep layer $V$ neurons was typical in many injections as shown here. (C) An example injection at a more posterior site with clear superficial and deep labeling of cortical neurons. Histological references are modified from Paxinos and Franklin, 2004 with coordinates relative to bregma shown $(\mathrm{mm})$. Scale bars: $0.5 \mathrm{~mm}$ (A-C).

examined with paired injections of tracers and inspection of the resulting tissue as we have done here, we note that this model of connectivity also predicts that there should be a well-defined covariance structure in the afferent inputs to the striatum. To examine whether there was evidence of such correlations in our dataset we computed the pairwise correlations between annotated brain regions for all injection sites (Figure 2B).

As expected, there is a large distribution of correlation scores with the majority of correlations being weak and non-significant (FigureA1 of Appendix). Nonetheless, comparison of the observed correlation values with the expected distribution from a random pattern reveals that our observed distribution is significantly shifted toward positive correlations (Figure A1B of Appendix; $p<0.0001$ ).

Examination of the distribution of significant correlations (Figure A1A of Appendix) revealed both significant positive correlations between specific brain areas and larger trends in the overall 

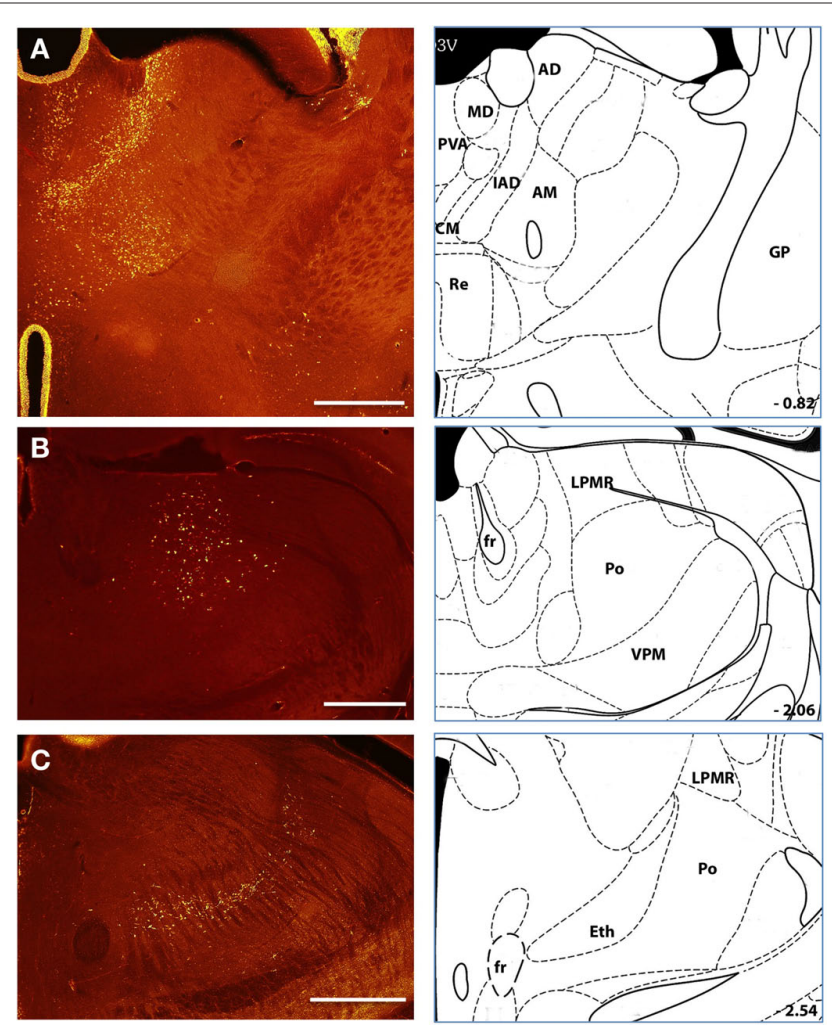

FIGURE 4 | Major sources of thalamostriatal projection Labeled neurons following retrograde tracer injections into the anterior $(A)$ and posterior $(B, C)$ aspect of the dorsal striatum. Anterior injection sites were associated with labeled cells throughout a wide range of thalamic nuclei $(\mathbf{A})$ including the PAV. MD. CM, IAD. AD, Re. AM., and LPMR (see text for details). Posterior injection sites predominantly labeled $\mathrm{PO}$ and Eth nuclei and PF nuclei at more medial injection sites (not shown). Histological references are modified from Paxinos and Franklin, 2004 with coordinates relative to bregma shown (mm). Scale bars: $0.5 \mathrm{~mm}$.

distribution of labeled areas. For example, we found that the PO nucleus of the thalamus and the border of the secondary somatosensory cortex (S2-GI) were highly correlated $\left(r^{2}=0.49\right)$, whereas the PF nucleus of the thalamus was correlated with labeling in the primary somatosensory cortex $\left(S 1 ; r^{2}=0.50\right)$. The medial dorsal nucleus of the thalamus (MD) was likewise correlated with frontal and motor cortical areas $\left(\mathrm{M} 1-\mathrm{Fr} 3 ; r^{2}=0.48\right)$. In addition to the correlations between thalamostriatal and corticostriatal projections we also found significant correlations between nigrostriatal and corticostriatal projections. For example, labeling intensity in A10 was significantly correlated with labeling of the prefrontal cortex (PFC; $\left.r^{2}=0.46\right)$, whereas A9 labeling was anticorrelated $\left(r^{2}=-0.65\right)$.

The above correlations suggested the presence of potentially important functional convergence across striatal input pathways. However, careful examination of the labeled areas and correlations indicated that the convergence of inputs is related to their position along the anterior-posterior and medial-lateral neuraxes in addition to their putative functional overlap. For example, the more medial A10 was correlated strongly with medial cortical and thalamic nuclei, whereas the more lateral A9 was anti-correlated
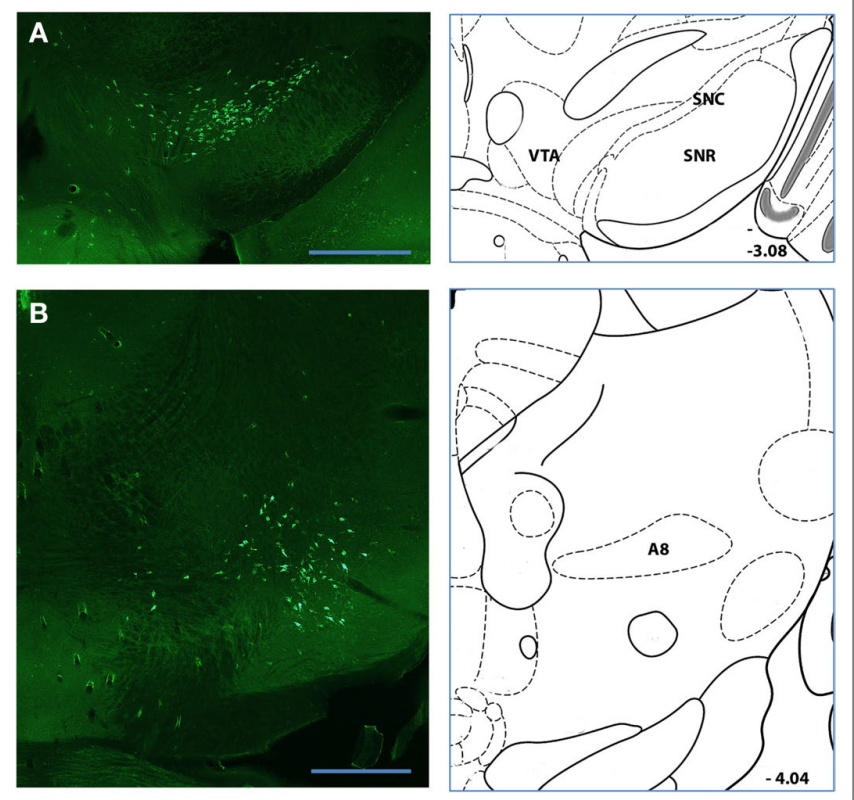

FIGURE 5 | Major sources of nigrostriatal projection (A,B) Labeled neurons following retrograde tracer injections into a posterior position of the dorsal striatum. Pronounced and intense somatic labeling was detected in putative dopaminergic neurons of the SNc (A) and retrorubral nucleus [A8; (C)]. Histological references are modified from Paxinos and Franklin. 2004 with coordinates relative to bregma shown $(\mathrm{mm})$. Scale bars: $0.5 \mathrm{~mm}$.

with those structures. The more lateral PO was likewise correlated more strongly with the more lateral secondary somatosensory cortex whereas the more medial PF was more strongly correlated with the more medio-dorsal primary somatosensory cortex. In addition, the distinctions along the anterior-posterior axis were particularly profound. The more anterior thalamic nuclei (e.g., AM) were strongly correlated with more frontal cortical regions (e.g., PFC; $\left.r^{2}=0.80\right)$ and anti-correlated with posterior thalamic nuclei and parietal cortical regions. Further, the BLA correlated strongly with a posterior localized thalamic nucleus, $\mathrm{PO}\left(r^{2}=0.57\right)$, by contrast to the CEA which was highly correlated with a more anterior thalamic nucleus, $\operatorname{RE}\left(r^{2}=0.68\right)$.

To evaluate the relative contribution of position within the neuraxis upon the structure of the correlations between labeled brain areas we calculated the eigen decomposition of the covariance matrix (Figure A2 of Appendix). Plotting the eigenvalues revealed, as expected, that there are many components of the covariance structure necessary to account for the dataset (Figure A2A of Appendix). Nonetheless, we find that the first two eigenvalues were significantly separated from the rest of the distribution and accounted for $41.6 \%$ of the variance. The corresponding eigenvectors should reflect the relative weighting given to individual brain areas necessary to generate the observed covariance. So we asked whether the weightings of the dominant eigenvectors reflected the position of labeled nuclei along the neuraxis. For clarity we used the estimated centroid position of our annotations of thalamic nuclei. We found that indeed the weightings in the first two eigenvectors are proportional to the position of the thalamic nuclei along both the anterior-posterior and medial-lateral neuraxes (Figures A2B,C 

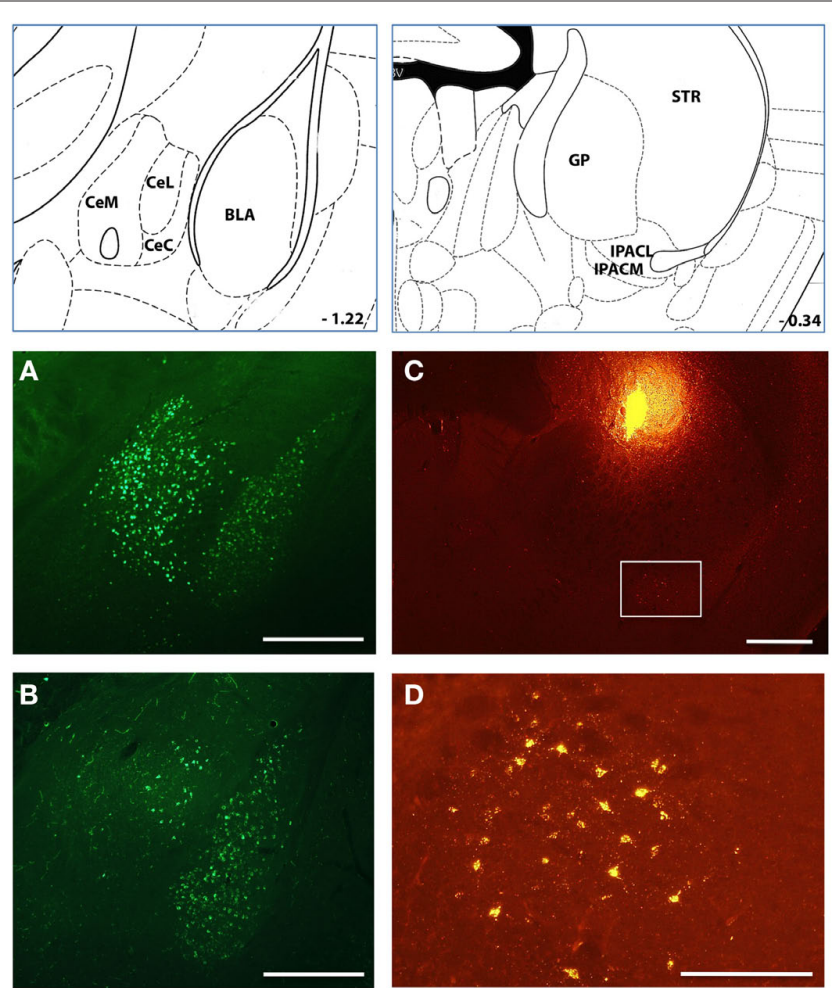

FIGURE 6 |A prominent amygdalostriatal projection in mice Injections of retrograde tracer into many sites throughout the dorsomedial striatum resulted in substantial labeling of cells in the amygdala [both central and basolateral nuclei; (A,B)] and the extended amygdala [IPAC; (C)]. (D) Inset of from $\mathrm{c}$ is shown in the image in (D). Histological references are modified from Paxinos and Franklin, 2004 with coordinates relative to bregma shown (mm). Scale bars; $0.5 \mathrm{~mm}$ (A-C) 0,2 $\mathrm{mm}$ (D)
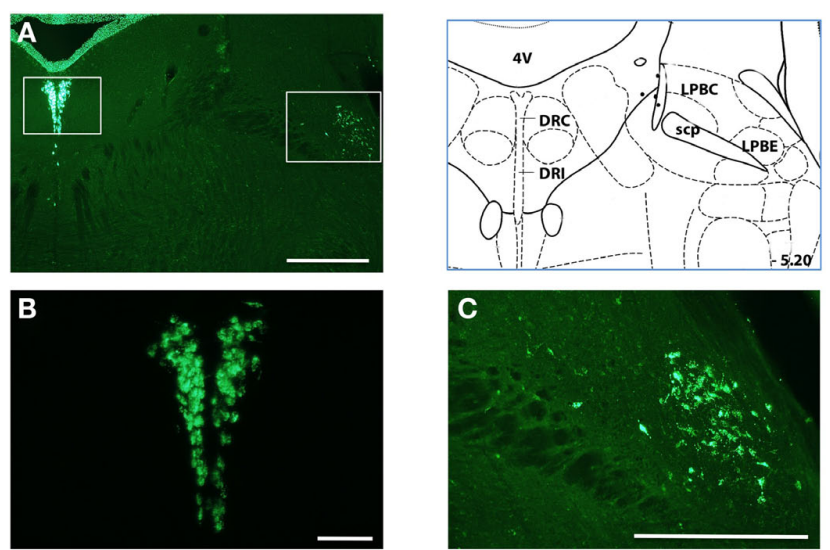

FIGURE 7 | Projections from the dorsal brainstem to the dorsal striatum (A) Injections of retrograde tracer produced less prominent but readable labeling of neurons in the dorsal brainstem. Left inset is region shown in (B); Right inset is region shown in (C). (B) inset from panel a shows putative serotonergic neurons in the dorsal raphe. Neurons of the medial raphe were also commonly labeled (not shown). More lateral nuclei were also occasionally observed. (C) intense labeling of the LP6E is shown in the inset from panel (A). Histological references are modified from Paxinos and Franklin, 2004 with coordinates relative to bregma shown (mm). Scale bars: $0.5 \mathrm{~mm}$ (A,C) $0.2 \mathrm{~mm}$ (B)

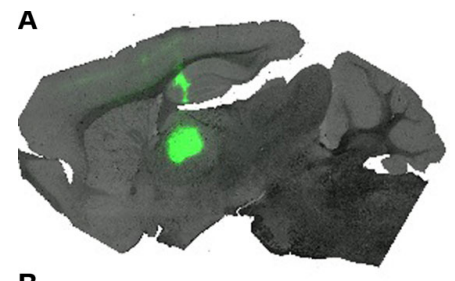

B
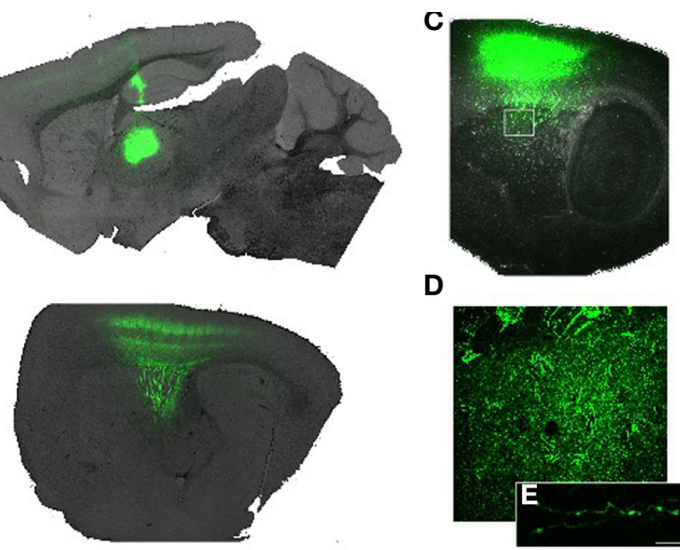

D

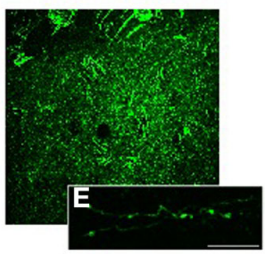

FIGURE 8 | Anterograde tracing from posterior thalamic nuclei (A) Parasagittal plane ( $1.5 \mathrm{~mm}$ lateral of bregma) showing the infection site in the posterior and lateral region of the thalamus. (B) A more lateral $(\sim 2.6 \mathrm{~mm}$ lateral) parasagittal plane showing extensive projection of thalamocortical axons into primary somatosensory cortex. (C) At the lateral extreme of the striatum ( $\sim 3 \mathrm{~mm}$ lateral) careful examination of the image reveals extensive ramification of axons in the striatum. Box indicates region magnified in (D). (D) Magnified image showing diffuse and tortuous labeled axons. (E) Confocal image showing two axons studded with varicosities. Scale bar $10 \mathrm{~m}$.

of Appendix), consistent with the claim that position along these axes was the most important determinant of their postsynaptic targets in the dorsal striatum.

\section{Organization of inputs along the medial-lateral axis of the dorsal striatum}

As described above, our analysis of the pairwise correlations amongst afferent input structures to the dorsal striatum indicated that position along the medial-lateral axis and the anterior-posterior axis were important determinants of their postsynaptic targets in the striatum. However, even though we selected fluorescent beads for their useful property minimal diffusion away from the injection site and uptake by actively cycling axon terminals (Vercelli et al., 2000), it is still difficult to precisely identify the site of injection and to rule out a biased diffusion or in homogeneous uptake of tracer across injections. Thus, to confirm our inferences from the correlation structure of all cases, we examined injections in which beads with distinct fluorophores were targeted to positions separated along the medial-lateral axis in the same subject.

From simultaneous injection of two tracers we found that the medial to lateral separation of inputs is maintained throughout the neuraxis. In the frontal cortex we found the infralimbic cortex was labeled clearly from the medial injection site whereas agranular motor cortex was labeled from the lateral injection site (Figure 9A). The separation was particularly clear in the ventral midbrain. Whereas nearly overlapping injection sites with two different fluorescent beads labeled intermingled (and largely non-overlapping) sets of dopaminergic neurons, injection sites with a significant medial to lateral separation labeled non-overlapping collections of midbrain dopaminergic neurons (Figure 9C). Retrograde labeling in the thalamus also showed a robust medial to lateral separation and a clear anterior to posterior separation (Figure 9B). 


\section{Organization of inputs along the anterior-posterior axis of the dorsal striatum}

We were particularly interested in the posterior striatum because it has been relatively less studied and as we have confirmed in mice, is known to receive extensive projections from sensory regions of cortex and the sensory thalamus (Figure 2). As noted above, examination of the correlations between retrogradely labeled input structures suggested a surprisingly clear separation between anterior and posterior striatal injection sites. The divergence of labeling we detected between anterior and posterior injection sites was surprising based upon studies in other mammals. In the mouse as in many mammals, the sensory and motor areas of the neocortex are segregated along the anterior-posterior axis. This is also the case in the primate, but an elegant series of studies demonstrated a robust convergence of functionally related sensory and motor structures in the striatum despite this separation along the anterior-posterior axis (Ragsdale and Graybiel, 1990; Parthasarathy et al., 1992; Flaherty and Graybiel, 1995; Parthasarathy and Graybiel, 1997). In the rat sensory and motor projections to the striatum are only partially overlapping (Hoffer and Alloway, 2001; Alloway et al., 2006) even though sensory and motor areas at the same position in the anterior-posterior axis may converge more substantially (Ramanathan et al., 2002). Thus, we sought to examine the projections from the well characterized sensory and motor cortex of the vibrissa system in the mouse.
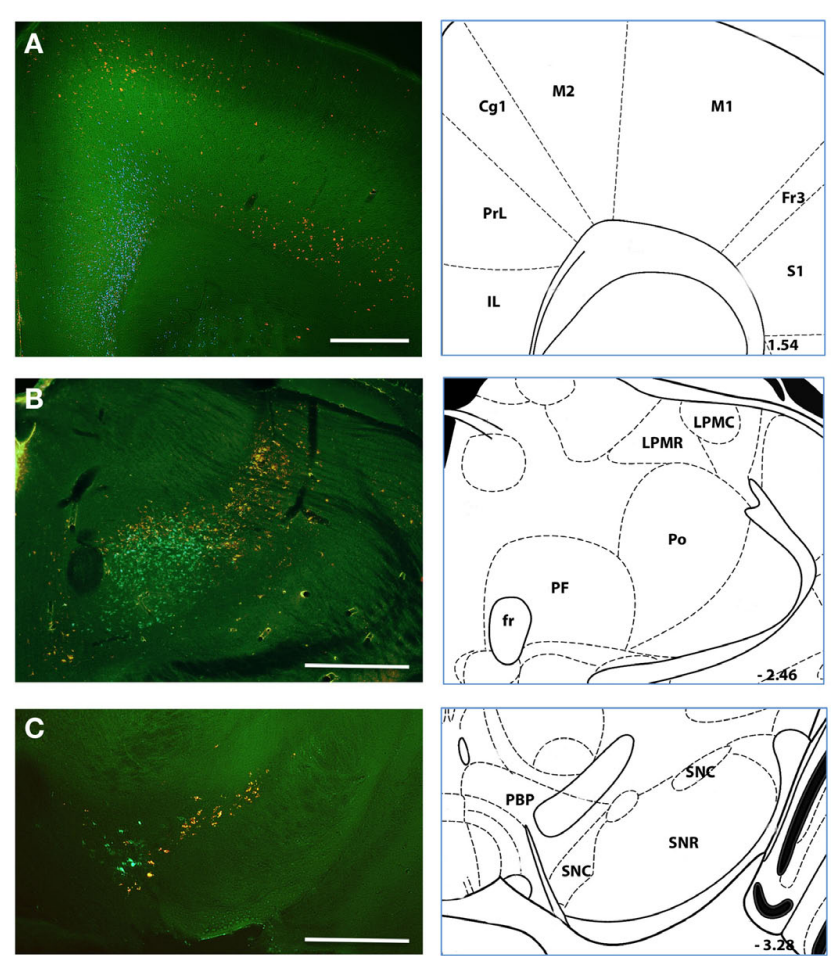

FIGURE 9 | Simultaneous injection of medial and lateral striatum Representative case in which injections of retrograde tracer were made in both a medial (green) and lateral (red) position in the dorsal striatum. Labeling in (A-C) shows the organization of labeled somata along the neuraxis from frontal cortices (A) to thalamus (B) to midbrain (C). Histological references are modified from Paxinos and Franklin, 2004 with coordinates relative to bregma shown $(\mathrm{mm})$. Scale bars: $0.5 \mathrm{~mm}$.
The primary motor and primary somatosensory areas of the vibrissa system were labeled by infection with a virus driving the over expression of fluorescent protein. Injections into somatosensory cortex led to a focal projection into the dorsolateral extreme of the posterior striatum (Figure 10B). By contrast, injections into primary motor cortex revealed a substantial arborization of axons in the anterior striatum and an axonal projection extending along the posterior axis (Figure 10A). While this extension along the posterior axis indeed partially overlapped with sensory axons as seen in rat (Hoffer and Alloway, 2001), the majority of the projection was several hundred microns more anterior to the sensory axons and largely non-overlapping. This observation was consistent with our retrograde labeling (Figure 2). Further, we found more prominent ipsilateral projections as opposed to contralateral projections, especially following infection of the somatosensory cortex that was again consistent with retrograde tracing.

\section{DISCUSSION}

We performed a series of injections of retrograde tracer into a range of positions within the dorsal striatum of the mouse. As the mouse becomes an increasingly popular model organism for
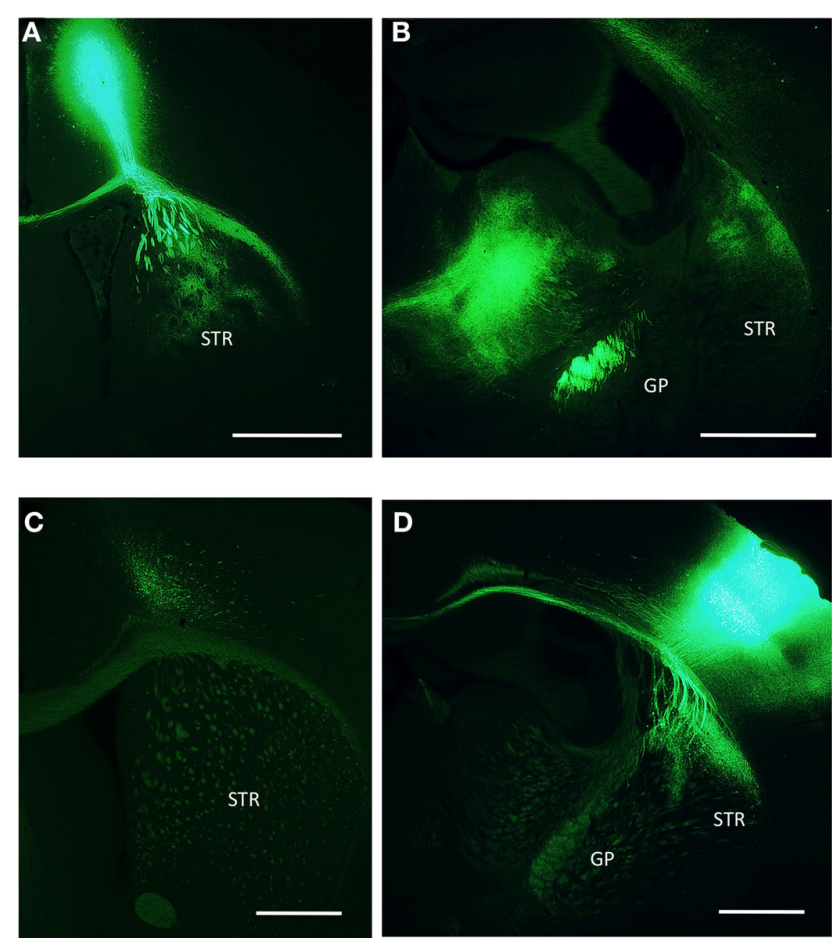

FIGURE 10 |Anterograde tracing from M1 and S1 cortices (A) Infection site in the primary motor cortex for the vibrissa. Descending axons ramify both locally within the same coronal plane of the striatum as well as fibers running caudally along the outer edge of the dorsal striatum. (B) Axons from the primary motor cortex are visible at a posterior coronal plane terminating densely in the thalamus and the dorsolateral striatum.

(C) Image taken from the same anterior coronal plane as a following infection of the primary somatosensory cortex. (D) Posterior plane shows infection site in the primary somatosensory cortex and local projection of axons into the dorsolateral striatum. GP, globus pallidas; STR, striatum. Scale bars: $1 \mathrm{~mm}$ 
neuroscientists, and a tractable model for the exploration of diseases of the basal ganglia, we found it increasingly important to confirm the homology between neuroanatomy in the mouse and the wealth of anatomical data in the rat. As a preliminary move in this direction we have begun to characterize the distribution of inputs to the dorsal striatum. The dorsal striatum is a massive structure receiving converging inputs from regions distributed through the telencephalon and diencephalon. As a consequence we have necessarily provided only a partial sampling of the potential loci for injection of retrograde tracers. However, we have applied an analysis of retrograde labeling using the covariance of labeling across multiple experiments that to our knowledge had not been applied to such data previously and may prove to be a useful approach for future studies (Figures 2, A1 and A2 of Appendix). Our analysis provided suggestive evidence for the convergence of projections from specific nuclei across the nigro-, cortico-, thalamo-, and amygdalo- striatal pathways. We also have provided some quantitative evidence that the position of an afferent structure along the anterior-posterior and medial-lateral neuraxes is the principle determinant of the termination field within the dorsal striatum, consistent with data in the rat (Paxinos, 2004). These observations from analysis of our dataset were confirmed by both dual injections of retrograde tracer (Figure 9) and anterograde tracing (Figure 10). Finally, we have provided supporting evidence for projections from the amygdala and raphe nuclei to dorsal striatum that were first identified in rat, but have received less attention in the years since their discovery.

\section{THE THALAMOSTRIATAL PROJECTION}

As expected, and described above, we find that there are deep similarities between the extensive literature on the canonical striatal inputs from the thalamus, ventral midbrain dopamine neurons and deep layer cortical neurons in the rat and our data in mice. Here we have provided some refinement of the details and interpretation of these projections when compared with the canonical descriptions. For example, in the thalamostriatal projection we found that nuclei outside of the intralaminar nuclei of the thalamus appear to make a significant contribution to the projection into the posterior striatum. This observation was discovered by retrograde labeling of PO nuclei of the thalamus, controlled for by the implantation of cannulae to prevent leakage along the injection tract, and confirmed through anterograde tracing following infection of the posterior and ventral posterior thalamic nuclei. The PO nucleus receives primary sensory afferents from brainstem nuclei (Paxinos, 2004) and the Eth nucleus additionally receives feedback from the motor cortex via zona incerta (Barthó et al., 2002). This strongly suggests that in the mouse and the rat the thalamostriatal projection, like the corticostriatal projection, carries both primary sensory and motor information in addition to associative information.

\section{THE NIGROSTRIATAL PROJECTION}

We found that the nigrostriatal projection is topographically organized along a medial-lateral axis and includes projections from throughout the anterior-posterior extent (extending from areas A8-A10) of the dopaminergic cell bodies in the ventral midbrain. This medial-lateral organization was associated with the surprising observation that a significant component of the dorsal nigrostriatal projection originated from somata located at the anteromedial surface of the $\mathrm{SNr}$ - a position consistent with the localization of the VTA (Figure 5). We also consistently observed large labeled somata in the midbrain that was dorsal and posterior to the SNc, consistent with the retrorubral field (A8; Figure 5). Notably absent, however, were "displaced" dopamine neurons within or along the ventral surface of the SNr (Gerfen et al., 1987; Prensa and Parent, 2001). Prior work demonstrated that displaced dopamine neurons form a specific projection to very dorsal and lateral regions of the striatum (Gerfen et al., 1987) that may have been under sampled in our injections.

Based upon retrograde and anterograde labeling in the primate it has been previously suggested that dopaminergic neurons may play a critical role in transferring information between the medial and lateral striatum (Haber et al., 2000; Haber, 2003). Although future experiments will be required to determine whether such a "spiral" structure is present in the rodent brain (Yin et al., 2008; Belin et al., 2009), our data are consistent with a topographical organization that could subserve a similar function.

\section{THE CORTICOSTRIATAL PATHWAY}

Finally, by using injection sites distributed over a range of sites along the anterior-posterior and medial-lateral axes of the dorsal striatum we were able to confirm the medial-lateral gradient of corticostriatal projections first elucidated in the rat and primate. The most parsimonious account of our data is that the topography of striatal inputs predominantly maintains the topographic organization of the neocortex and thalamus. Consistent with this interpretation we also find that there is a distinct anterior-posterior gradient present in both the thalamostriatal and corticostriatal projections. Although it has received less attention as an important axis of functional differentiation, recent results suggest that the anterior and posterior striatum have dissociable behavioral effects (Tricomi et al., 2009; Shiflett et al., 2010). Indeed, our analysis of the correlation between labeled thalamic nuclei suggests that the anterior-posterior axis may be a more significant axis of differentiation in the dorsal striatum than the medial-lateral axis (Figure A2 of Appendix).

Whereas in the monkey there appears to be strong convergence of functionally related, but topographically distant areas in the corticostriatal projection, our results suggest that for the majority of axons in the corticostriatal projection these topographic separations are maintained in the mouse. This observation is consistent with the distribution of the projection from the vibrissa primary motor and vibrissa primary sensory cortex in the rat where less than $5 \%$ overlap in axonal projections has been observed (Hoffer and Alloway, 2001). However, even though the overlap of motor and sensory axons was an apparent minority of the labeling, the zones of overlap may be disproportionately important to the function of the dorsolateral striatum in the rat (Brown and Sharp, 1995), as has been proposed in the primate (Graybiel et al., 1994; Haber, 2003; Cohen and Frank, 2009; Haber and Calzavara, 2009). In rodents, an assessment of the functional importance of convergence and divergence in the corticostriatal and thalamostriatal projections is likely to be an important area of study for experiments in the future.

\section{NON-CANONICAL STRIATAL PROJECTIONS}

In addition to these core features of the macrocircuitry of the striatum that were common across a wide range of vertebrates (Reiner et al., 1998; Tepper et al., 2007), our injections also identified 
a collection of nuclei and brain regions that have been previously observed, but are not commonly included in the canonical basal ganglia circuit. Most strikingly, we found strong labeling of the amygdala, the extended amygdala, and the raphe nuclei. The projection of serotonergic neurons to the dorsal striatum has received some attention (Di Matteo et al., 2008), especially interesting due to its proposed role in mediating opponent learning (Daw et al., 2002; Doya, 2008). However, while projections from the amygdala to the ventral striatum have indeed received quite a lot of attention (Gray, 1999; Cardinal et al., 2002; Floresco et al., 2008; Belin et al., 2009; Humphries and Prescott, 2010), the projections to the dorsal striatum that were observed with anterograde tracing in the rat (Kelley et al., 1982), have received relatively less attention. To our surprise, in the mouse we regularly detected significant labeling of the amygdala following injections across a range of different positions within the dorsal striatum - with the notable exception of the most anterior and lateral injections (Figure 2). A previous study in the rat also demonstrated that the only territory where amygdala fibers were absent was in the rostrolateral striatum, consistent with our results (Kelley et al., 1982). The importance of the amygdalostriatal projection into dorsal striatum was further suggested by the observation that it is an evolutionarily ancient pathway (Martínez-García et al., 2002) present at least as far back as reptiles (Novejarque et al., 2004).

We suggest that the prominent amygdalostriatal projection to the dorsomedial striatum should be given some attention and perhaps inclusion in the canonical circuitry of the basal ganglia. Indeed, it has recently been demonstrated that activity in the amygdala is highly correlated with learning and reflects both value and error prediction signals that are tightly correlated with learning (Paton et al., 2006; Belova et al., 2008). Given the critical requirement of the dorsal striatum in the acquisition and expression of goal-directed instrumental conditioning and the central role that error prediction signals are thought to play in such learning (Schultz, 2006), the amygdalostriatal projection may be an important component of an extended learning circuit involving the hippocampus, dorsal striatum, and amygdala (McDonald et al., 2007).

\section{LIMITATIONS OF OUR DATASET}

We selected fluorescent latex microspheres because of their lack of diffusion away from the injection site and high signal-to-noise ratio compared to other approaches (Vercelli et al., 2000). Nonetheless, it is currently not possible to accurately estimate the number of beads injected or differences in the efficiency of uptake by the surrounding axons. Our analysis of the covariance of labeling is more susceptible to another problem: full sampling of a structure as large as the dorsal striatum is impractical. While we have necessarily under sampled the complete distribution of inputs to the striatum, two features of our study are important. One, there was a lack of prior datasets covering multiple striatal subregions in the mouse. Two, our analysis provides a general method that could be applied to future datasets or accommodate additional data and more complete coverage of the dorsal striatum.

Medium spiny neurons contain upwards of 10,000 synapses in the rat, with approximately a quarter of all synapses being symmetrical synapses (Wilson, 2007). Single cell labeling studies and paired recordings have indicated that many of the symmetrical synapses found on MSNs arise from local GABAergic interneurons that have extensive, locally ramifying axonal arborizations (Tepper et al., 2008). In addition, elegant single cell labeling studies have discovered a feedback pathway from the GP to the striatum (Bevan et al., 1998). Neither of these projections was reported in our summary data. For local connectivity we regularly observed labeled neurons locally within the dorsal striatum (e.g., Figure 3C), however, it was unclear how to quantify this labeling or if one could confidently distinguish direct labeling due to pressure injection, leakage into the underlying GP, or uptake by local axon collaterals. Thus, we have left these projections out of our analysis. By contrast, with a single exception we did not detect labeling of neurons in the GP in our injections except in cases where the injection site may have had minor overlap with the GP. It remains to be seen in future work whether the absence of a detectable pallidostriatal projection was an artifact of our technique or reflects an actual difference between rats and mice in the organization of the basal ganglia.

\section{FUNCTIONAL ARCHITECTURE OF THE MOUSE STRIATUM}

Both our study and many previous studies in the rat (Paxinos, 2004) have provided evidence for a well-defined topographic segregation along the medial-lateral and anterior-posterior axis. However, this observation of a segregation of pathways has also raised questions about how activity in anatomically distinct regions of cortex, thalamus, and striatum are coordinated during learning. Perhaps the nigrostriatal pathway mediates this integration (Haber, 2003) via thalamocortical loops (Haber and Calzavara, 2009). A complimentary possibility is that extensive intracortical projections may play an important role in rodents. A less studied possibility is that local inhibitory interactions, present throughout the basal ganglia (Tepper et al., 2007) and prominent in the striatum (Wilson, 2007), play a critical role in coordinating activity during learning. Our examination of the correlation structure of inputs to the striatum suggests a diverse collection of neuronal groups with idiosyncratic collections of afferents. These diverse target areas, as has been occasionally noted in the literature (Brown, 1992), may allow for a combinatorial map of cortical output in the striatum. Thus, local inhibitory interactions may be sufficient to mediate interactions between and across multiple sensory and motor modalities, rather than or in addition to interactions within corticothalamic loops.

Our study of the striatum in mouse has provided a helpful step in the goal of combining the power of a genetic model organism and circuit anatomy in the basal ganglia. Future work will be required to both further complete a description of the macrocircuitry of the mouse as well as to enhance the specific description of the microstructure within the corticostriatal and thalamostriatal projections. Indeed, an exciting prospect for the future is the use of tracing techniques for studies of macrocircuitry in combination with the use of optical and genetic approaches for the elucidation of microcircuitry with cellular resolution.

\section{ACKNOWLEDGMENTS}

Weixing X. Pan, Tianyi Mao, and Joshua T. Dudman performed the experiments. Joshua T. Dudman and Weixing X. Pan analyzed the data and wrote the paper. We are indebted to the excellent technical support we received from Amy Hu, Alma Arnold, and Susan Michael. We thank Alla Karpova for a careful reading of the manuscript. 


\section{REFERENCES}

Albin, R. L., Young, A. B., and Penney, J. B. (1989). The functional anatomy of basal ganglia disorders. Trends Neurosci. 12, 366-375.

Alloway, K. D., Lou, L., NwabuezeOgbo, F., and Chakrabarti, S. (2006). Topography of cortical projections to the dorsolateral neostriatum in rats: multiple overlapping sensorimotor pathways. J. Comp. Neurol. 499, 33-48.

Alloway, K. D., Mutic, J. J., Hoffer, Z. S., and Hoover, J. E. (2000). Overlapping corticostriatal projections from the rodent vibrissal representations in primary and secondary somatosensory cortex. J. Comp. Neurol. 428, 51-67.

Balleine, B. W., Liljeholm, M., and Ostlund, S. B. (2009). The integrative function of the basal ganglia in instrumental conditioning. Behav. Brain Res. 199, 43-52.

Barroso-Chinea, P., Castle, M., Aymerich, M. S., and Lanciego, J. L. (2008). Expression of vesicular glutamate transporters 1 and 2 in the cells of origin of the rat thalamostriatal pathway. J. Chem. Neuroanat. 35, 101-107.

Barthó, P., Freund, T. F., and Acsády, L. (2002). Selective GABAergic innervation of thalamic nuclei from zona incerta. Eur. J. Neurosci. 16, 999-1014.

Belin, D., Jonkman, S., Dickinson, A., Robbins, T.W., and Everitt, B. J. (2009). Parallel and interactive learning processes within the basal ganglia: relevance for the understanding of addiction. Behav. Brain Res. 199, 89-102.

Belova, M. A., Paton, J. J., and Salzman, C. D. (2008). Moment-to-moment tracking of state value in the amygdala. $J$. Neurosci. 28, 10023-10030.

Bernardo, K. L., and Woolsey, T.A. (1987). Axonal trajectories between mouse somatosensory thalamus and cortex. J. Comp. Neurol. 258, 542-564.

Bevan, M. D., Booth, P. A., Eaton, S. A., and Bolam, J. P. (1998). Selective innervation of neostriatal interneurons by a subclass of neuron in the globus pallidus of the rat. J. Neurosci. 18, 9438-9452.

Brazhnik, E., Shah, F., and Tepper, J. M. (2008). GABAergic afferents activate both GABAA and GABAB receptors in mouse substantia nigra dopaminergic neurons in vivo. J. Neurosci. 28, 10386-10398.

Brown, L. L. (1992). Somatotopic organization in rat striatum: evidence for a combinational map. Proc. Natl. Acad. Sci. U.S.A. 89, 7403-7407.

Brown, L. L., Hand, P. J., and Divac, I. (1996). Representation of a single vibrissa in the rat neostriatum: peaks of energy metabolism reveal a distributed functional module. Neuroscience 75, 717-728.

Brown, L. L., and Sharp, F. R. (1995). Metabolic mapping of rat striatum: somatotopic organization of sensorimotor activity. Brain Res. 686, 207-222.

Brown,L.L.,Smith, D.M., and Goldbloom, L.M. (1998). Organizing principles of cortical integration in the rat neostriatum: corticostriate map of the body surface is an ordered lattice of curved laminae and radial points. J. Comp. Neurol. 392, 468-488.

Cardinal, R. N., Parkinson, J. A., Hall, J., and Everitt, B. J. (2002). Emotion and motivation: the role of the amygdala, ventral striatum, and prefrontal cortex. Neurosci. Biobehav. Rev. 26, 321-352.

Cepeda, C., Hurst, R. S., Calvert, C. R., Hernández-Echeagaray, E., Nguyen, O. K., Jocoy, E., Christian, L. J., Ariano, M. A., and Levine, M. S. (2003). Transient and progressive electrophysiological alterations in the corticostriatal pathway in a mouse model of Huntington's disease. J. Neurosci. 23, 961-969.

Cohen, M. X., and Frank, M. J. (2009). Neurocomputational models of basal ganglia function in learning, memory and choice. Behav. Brain Res. 199, 141-156.

Daw, N. D., Kakade, S., and Dayan, P. (2002). Opponent interactions between serotonin and dopamine. Neural. Netw. 15, 603-616.

Deniau, J. M., Mailly, P., Maurice, N., and Charpier,S. (2007). The pars reticulata of the substantia nigra: a window to basal ganglia output. Prog. Brain Res. 160, 151-172.

Di Matteo, V., Pierucci, M., Esposito, E., Crescimanno, G., Benigno, A., and Di Giovanni, G. (2008). Serotonin modulation of the basal ganglia circuitry: therapeuticimplication for Parkinson's disease and other motor disorders. Prog. Brain Res. 172, 423-463.

Doya, K. (2008). Modulators of decision making. Nat. Neurosci. 11, 410-416.

Erro, E., Lanciego, J. L., and GiménezAmaya, J. M. (1999). Relationships between thalamostriatal neurons and pedunculopontine projections to the thalamus: a neuroanatomical tracttracing study in the rat. Exp. Brain Res. 127, 162-170.

Erro, E. M., Lanciego, J. L., Arribas, J., and Giménez-Amaya, J.M. (2001).Striatal input from the ventrobasal complex of the rat thalamus. Histochem. Cell Biol. $115,447-454$.

Erro, E. M., Lanciego, J. L., and GimenezAmaya, J. M. (2002). Re-examination of the thalamostriatal projections in the rat with retrograde tracers. Neurosci. Res. 42, 45-55.

Flaherty,A.W., and Graybiel,A.M. (1991). Corticostriatal transformations in the primate somatosensory system. Projections from physiologically mapped body-part representations J. Neurophysiol. 66, 1249-1263.

Flaherty,A.W., and Graybiel, A. M. (1995) Motor and somatosensory corticostriatal projection magnifications in the squirrel monkey. J. Neurophysiol. 74 2638-2648.

Floresco, S. B., St Onge, J. R., GhodsSharifi, S., and Winstanley, C. A (2008). Cortico-limbic-striatal circuits subserving different forms of costbenefit decision making. Cogn. Affect. Behav. Neurosci. 8, 375-389.

Gerfen, C. R. (1984). The neostriata mosaic: compartmentalization of corticostriatal input and striatonigral output systems. Nature 311, 461-464.

Gerfen, C. R. (1989). The neostriatal mosaic: striatal patch-matrix organization is related to cortical lamination. Science 246, 385-388.

Gerfen, C. R. (1992). The neostriatal mosaic: multiple levels of compartmental organization in the basal ganglia. Annu. Rev. Neurosci. 15, 285-320.

Gerfen, C. R., Herkenham, M., and Thibault, J. (1987). The neostriatal mosaic: II. Patch- and matrix-directed mesostriatal dopaminergic and nondopaminergic systems. J. Neurosci. 7 3915-3934.

Goldman, P. S., and Nauta, W. J. (1977) An intricately patterned prefrontocaudate projection in the rhesus monkey. J. Comp. Neurol. 72, 369-386.

Gray, T.S. (1999). Functional and anatomical relationships among the amygdala, basal forebrain, ventral striatum, and cortex. An integrative discussion. Ann N. Y. Acad. Sci. 877, 439-444.

Graybiel, A. M., Aosaki, T., Flaherty, A. W., and Kimura, M. (1994). The basal ganglia and adaptive motor control. Science 265, 1826-1831.

Haber, S. N. (2003). The primate basal ganglia: parallel and integrative networks. J. Chem. Neuroanat. 26, 317-330.

Haber, S. N., and Calzavara, R. (2009). The cortico-basal ganglia integrative network: the role of the thalamus. Brain Res. Bull. 78, 69-74.

Haber, S. N., Fudge, J. L., and McFarland, N. R. (2000). Striatonigrostriatal pathways in primates form an ascending spiral from the shell to the dorsolateral striatum. J. Neurosci. 20 2369-2382.

Hersch, S. M., and White, E. L. (1982) A quantitative study of the thalamocortical and other synapses in layer IV of pyramidal cells projecting from mouse SmI cortex to the caudateputamen nucleus. J. Comp. Neurol. 211, 217-225.
Hoffer, Z. S., and Alloway, K. D. (2001) Organization of corticostriatal projections from the vibrissal representations in the primary motor and somatosensory cortical areas of rodents. J. Comp. Neurol. 439, 87-103.

Hoffer, Z. S., Arantes, H. B., Roth, R. L. and Alloway, K. D. (2005). Functional circuits mediating sensorimotor integration: quantitative comparisons of projections from rodent barrel cortex to primary motor cortex, neostriatum, superior colliculus, and the pons. $J$. Comp. Neurol. 488, 82-100.

Hofstetter, K. M., and Ehret, G. (1992). The auditory cortex of the mouse: connections of the ultrasonic field. J. Comp. Neurol. 323, 370-386.

Humphries, M. D., and Prescott, T. J. (2010). The ventral basal ganglia, a selection mechanism at the crossroads of space, strategy, and reward. Prog. Neurobiol. 90, 385-417.

Ibáñez-Sandoval, O., Tecuapetla, F., Unal, B., Shah, F., Koós, T., and Tepper, J. M (2010). Electrophysiological and morphological characteristics and synaptic connectivity of tyrosine hydroxylaseexpressing neurons in adult mouse striatum. J. Neurosci. 30, 6999-7016.

Kelley, A. E., Domesick, V. B., and Nauta, W. J. (1982). The amygdalostriatal projection in the rat-an anatomical study by anterograde and retrograde tracing methods. Neuroscience 7, 615-630.

Malach, R., and Graybiel, A. M. (1986). Mosaic architecture of the somatic sensory-recipient sector of the cat's striatum. J. Neurosci. 6, 3436-3458.

Martínez-García, F., Martínez-Marcos, A., and Lanuza, E. (2002). The pallial amygdala of amniote vertebrates: evolution of the concept, evolution of the structure. Brain Res. Bull. 57, 463-469.

Mattiace, L. A., Baring, M. D., Manaye, K. F., Mihailoff, G. A., and German, D. C. (1989). Mesostriatal projections in $\mathrm{BALB} / \mathrm{c}$ and CBA mice: a quantitative retrograde neuroanatomical tracing study. Brain Res. Bull. 23, 61-68.

McDonald, R. J., King, A. L., Wasiak, T. D., Zelinski, E. L., and Hong, N. S. (2007). A complex associative structure formed in the mammalian brain during acquisition of a simple visual discrimination task: dorsolateral striatum, amygdala, and hippocampus. Hippocampus 17, 759-774

Mink, J. W. (1996). The basal ganglia: focused selection and inhibition of competing motor programs. Prog. Neurobiol. 50, 381-425.

Miura, M., Saino-Saito, S., Masuda, M. Kobayashi, K., and Aosaki, T. (2007). Compartment-specific modulation of GABAergic synaptic transmission by mu-opioid receptor in the mouse 
striatum with green fluorescent protein-expressing dopamine islands. $J$. Neurosci. 27, 9721-9728.

Novejarque, A., Lanuza, E., and Martínez-García, F. (2004). Amygdalostriatal projections in reptiles: a tract-tracing study in the lizard Podarcis hispanica. J. Comp. Neurol. 479, 287-308.

Packard, M. G., and Knowlton, B. J. (2002). Learning and memory functions of the Basal Ganglia. Annu. Rev. Neurosci. 25, 563-593.

Parthasarathy, H. B., and Graybiel, A. M. (1997). Cortically driven immediate-early gene expression reflects modular influence of sensorimotor cortex on identified striatal neurons in the squirrel monkey. J. Neurosci. 17, 2477-2491.

Parthasarathy, H. B., Schall, J. D., and Graybiel,A. M. (1992). Distributed but convergent ordering of corticostriatal projections: analysis of the frontal eye field and the supplementary eye field in the macaque monkey. J. Neurosci. 12, 4468-4488.

Paton, J. J., Belova, M. A., Morrison, S. E., and Salzman, C. D. (2006). The primate amygdala represents the positive and negative value of visual stimuli during learning. Nature 439, 865-870.

Paxinos, G. (2004). The Rat Nervous System, 3rd Edn. San Diego: Elsevier Academic Press, 1309.

Paxinos, G., and Franklin, K. B. (2004). The Mouse Brain in Stereotaxic Coordinates, 2nd Edn. San Diego: Elsevier Academic Press, 100.

Porter, L. L., and White, E. L. (1983). Afferent and efferent pathways of the vibrissal region of primary motor cortex in the mouse. J. Comp. Neurol. 214, 279-289.

Prensa, L., and Parent, A. (2001). The nigrostriatal pathway in the rat: a single-axon study of the relationship between dorsal and ventral tier nigral neurons and the striosome/matrix striatal compartments. J. Neurosci. 21, 7247-7260.

Price, J. L. (1986). Subcortical projections from the amygdaloid complex. $A d v$. Exp. Med. Biol. 203, 19-33.

Ragsdale, C. W., and Graybiel, A. M. (1990). A simple ordering of neocortical areas established by the compartmental organization of their striatal projections. Proc. Natl. Acad. Sci. U.S.A. 87, 6196-6199.

Ramanathan, S., Hanley, J. J., Deniau, J.-M., and Bolam, J. P. (2002). Synaptic convergence of motor and somatosensory cortical afferents onto GABAergic interneurons in the rat striatum. $J$. Neurosci. 22, 8158-8169.

Reiner, A., Medina, L., and Veenman, C. L. (1998). Structural and functional evolution of the basal ganglia in vertebrates. Brain Res. Brain Res. Rev. 28, 235-285.

Schultz, W. (2006). Behavioral theories and the neurophysiology of reward. Annu. Rev. Psychol. 57, 87-115.

Shiflett, M. W., Brown, R. A., and Balleine, B. W. (2010). Acquisition and performance of goal-directed instrumental actions depends on ERK signaling in distinct regions of dorsal striatum in rats. J. Neurosci. 30, 2951-2959.

Smith, Y., Raju, D.V., Pare, J.-F., and Sidibe, M. (2004). The thalamostriatal system: a highly specific network of the basal ganglia circuitry. Trends Neurosci. 27, 520-527.

Tepper, J. M., Abercrombie, E. D., and Bolam, J.P. (2007). Gaba and the Basal Ganglia: From Molecules to Systems. Amsterdam: Elsevier, 339.

Tepper, J. M., Wilson, C. J., and Koós, T. (2008). Feedforward and feedback inhibition in neostriatal GABAergic spiny neurons. Brain Res. Rev. 58, 272-281.

Tlamsa, A. P., and Brumberg, J. C. (2010) Organization and morphology of thalamocortical neurons of mouse ventral lateral thalamus. Somatosens. Mot. Res. 27, 34-43.

Tricomi, E., Balleine, B.W., and O'Doherty, J. P. (2009). A specific role for posterior dorsolateral striatum in human habit learning. Eur. J. Neurosci. 29, 2225-2232.

Tulloch, I. F., Arbuthnott, G. W., and Wright, A. K. (1978). Topographical organization of the striatonigral pathway revealed by anterograde and retrograde neuroanatomical tracing techniques. J. Anat. 127, 425-441.

Usunoff, K. G., Schmitt, O., Itzev, D. E., Haas, S. J.-P., Lazarov, N. E., Rolfs, A., and Wree, A. (2009). Efferent projections of the anterior and posterodorsal regions of the medial nucleus of the amygdala in the mouse. Cells Tissues Organs (Print) 190, 256-285.

Vercelli, A., Repici, M., Garbossa, D., and Grimaldi, A. (2000). Recent techniques for tracing pathways in the central nervous system of developing and adult mammals. Brain Res. Bull. 51, 11-28.

White, E. L., and DeAmicis, R. A. (1977) Afferent and efferent projections of the region in mouse SmL cortex which contains the posteromedial barrel subfield. J. Comp. Neurol. 175, 455-482.

Wilson, C. J. (2007). GABAergic inhibition in the neostriatum. Prog. Brain Res. 160, 91-110.

Yin, H. H., Knowlton, B. J., and Balleine, B. W. (2005a). Blockade of NMDA receptors in the dorsomedial striatum prevents action-outcome learning in instrumental conditioning. Eur. J. Neurosci. 22, 505-512.
Yin, H. H., Ostlund, S. B., Knowlton, B. J., and Balleine, B. W. (2005b). The role of the dorsomedial striatum in instrumental conditioning. Eur. J. Neurosci. 22, 513-523.

Yin, H. H., Knowlton, B. J., and Balleine, B. W. (2006). Inactivation of dorsolateral striatum enhances sensitivity to changes in the action-outcome contingency in instrumental conditioning Behav. Brain Res. 166, 189-196.

Yin, H. H., Mulcare, S. P., Hilário, M. R. F., Clouse, E., Holloway, T., Davis, M. I., Hansson, A. C., Lovinger, D. M., and Costa, R. M. (2009). Dynamic reorganization of striatal circuits during the acquisition and consolidation of a skill. Nat. Neurosci. 12, 333-341.

Yin, H. H., Ostlund, S. B., and Balleine, B. W. (2008). Reward-guided learning beyond dopamine in the nucleus accumbens: the integrative functions of cortico-basal ganglia networks. Eur. J. Neurosci. 28, 1437-1448.

Conflict of Interest Statement: The authors declare that the research was conducted in the absence of any commercial or financial relationships that could be construed as a potential conflict of interest.

Received: 11 August 2010; accepted: 23 November 2010; published online: 27 December 2010.

Citation: Pan WX, Mao Tand Dudman JT (2010) Inputs to the dorsal striatum of the mouse reflect the parallel circuit architecture of the forebrain. Front. Neuroanat. 4:147. doi: 10.3389/fnana.2010.00147 Copyright @ 2010 Pan, Mao and Dudman. This is an open-access article subject to an exclusive license agreement between the authors and the Frontiers Research Foundation, which permits unrestricted use, distribution, and reproduction in any medium, provided the original authors and source are credited. 


\section{APPENDIX}
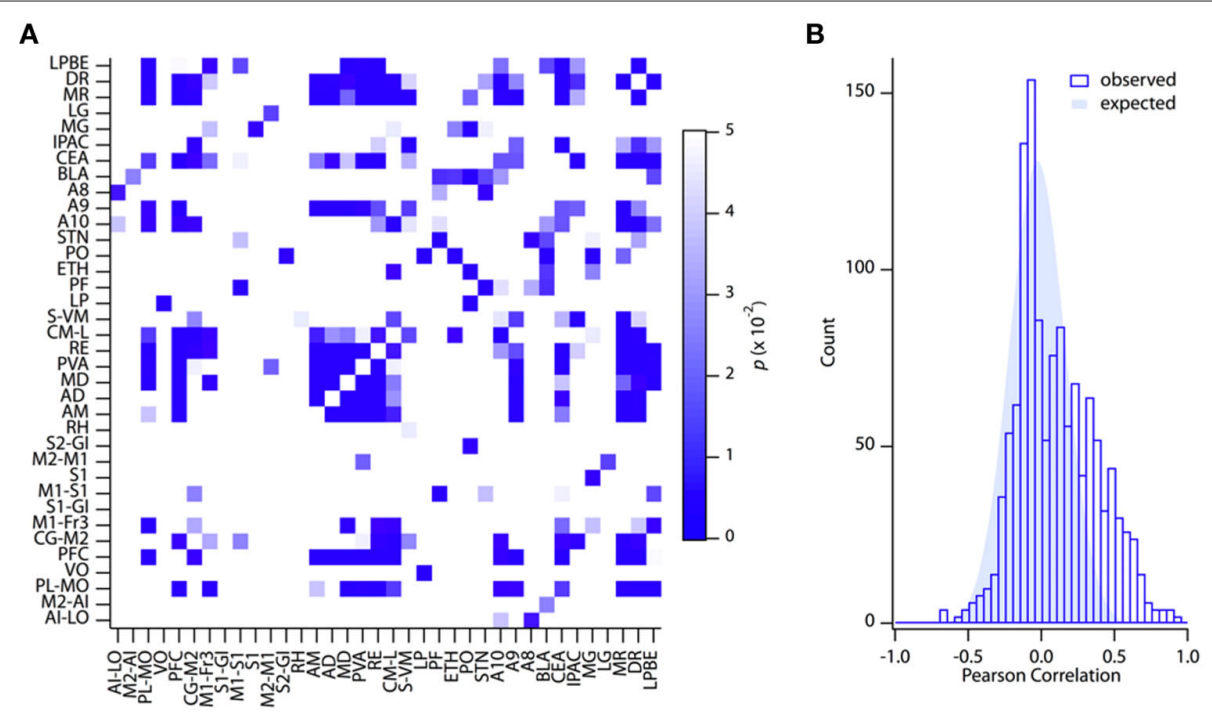

FIGURE A1 | Distribution and significance of pairwise correlations (A) Plot of estimated significance for each pairwise correlation value. For details about the plot please see corresponding plot in Figure 2 in the main text. (B) The observed distribution of all pairwise correlations is shown in open bars. Solid fill is a gaussian fit to the expected distribution of correlation scores for a random, equivalently scaled dataset.
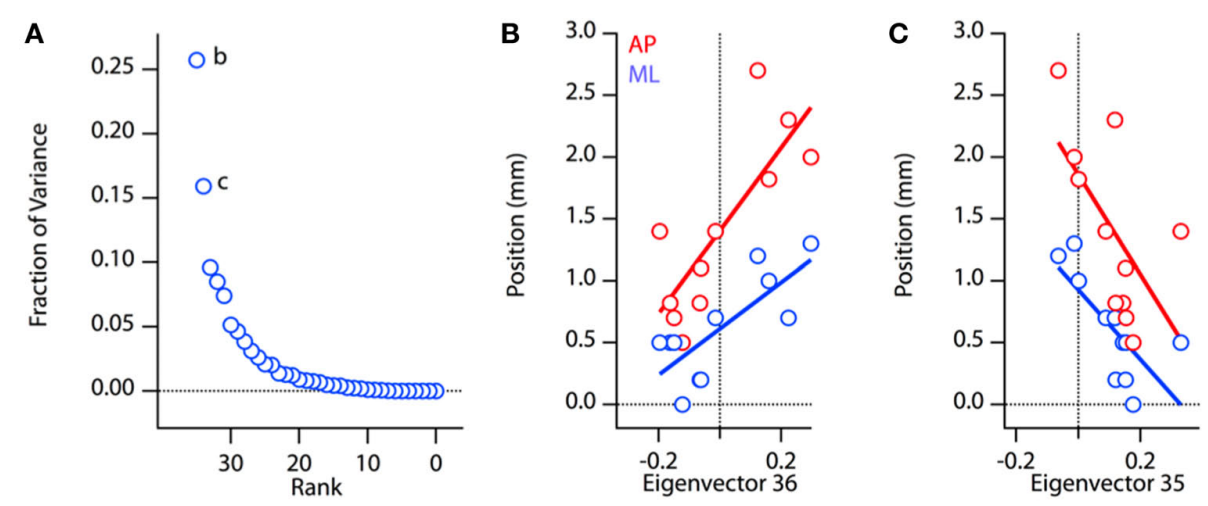

FIGURE A2 | Principal components analysis of retrograde labeling data (A) Rank ordered eigenvalues of the covariance matrix derived from retrograde labeling data in Figures 2 and Figure A1. (B,C) Correlation between the anterior-posterior (AP, red) and medial-lateral (ML, blue) position of annotated thalamic nuclei and the first (B) and second (C) eigenvectors (components) resulting from decomposition of the covariance matrix. Solid lines are best linear fits to the data. 